\title{
NÚMERO DE CASOS DE DIABETES TIPO 1 Y 2 DIAGNOSTICADOS EN AMAPÁ ENTRE 2007 Y 2012
}

\section{ARTÍCULO ORIGINAL}

SOUZA, Kauê de Melo', FACCO, Lucas², FECURY, Amanda Alves³, ARAÚJO, Maria Helena Mendonça de ${ }^{4}$, OLIVEIRA, Euzébio de ${ }^{5}$, DENDASCK, Carla Viana ${ }^{6}$, SOUZA, Keulle Oliveira da ${ }^{7}$, DIAS, Claudio Alberto Gellis de Mattos ${ }^{8}$

SOUZA, Kauê de Melo. Et al. Número de casos de diabetes tipo 1 y 2 diagnosticados en Amapá entre 2007 y 2012. Revista Científica Multidisciplinar Núcleo do Conhecimento. Año 05, Ed. 12, Vol. 01, págs. 18-26. Diciembre de 2020. ISSN: 2448-0959, Enlace de acceso:

https://www.nucleodoconhecimento.com.br/salud/numero-de-casos, DOI: 10.32749/nucleodoconhecimento.com.br/salud/numero-de-casos

\section{RESUMEN}

La diabetes mellitus es una sucesión de diferentes tipos de trastornos en el metabolismo que se caracterizan por causar una alta tasa de azúcar en la sangre. Debido a que es una enfermedad con factores genéticos tipo 1 la diabetes tiene como principal factor de riesgo la herencia, mientras que la diabetes tipo 2 además de estos factores, incluye obesidad, presión arterial alta, mala educación alimentaria y avance de la edad. Este estudio tiene como objetivo mostrar el número de casos

\footnotetext{
${ }^{1}$ Técnico Minero, como ministro del Instituto Federal de Amapá (IFAP).

2 Estudiante del Curso de Medicina de la Universidad Federal de Amapá (UNIFAP).

${ }^{3}$ Biomédica, Doctora en Enfermedades Tropicales, Profesora e investigadora del Curso Médico de la Universidad Federal de Amapá (UNIFAP).

${ }^{4}$ Médico, Profesor e investigador del Curso Médico de la Universidad Federal de Amapá (UNIFAP).

${ }^{5}$ Biólogo, Doctor en Enfermedades tópicas, Profesor e investigador del Curso de Educación Física de la Universidad Federal de Pará (UFPA).

${ }^{6}$ Teólogo, Doctor en Psicoanálisis, investigador del Centro de Investigación y Estudios Avanzados - CEPA.

${ }^{7}$ Sociólogo, Máster en Estudios Antrópicos en la Amazonía, Miembro del Grupo de Investigación "Laboratorio de Educación, Medio Ambiente y Salud" (LEMAS/UFPA).

${ }^{8}$ Biólogo, Doctor en Teoría e Investigación del Comportamiento, Profesor e investigador del Programa de Posgrado en Educación Profesional y Tecnológica (PROFEPT), Instituto Federal de Amapá (IFAP).
}

RC: 66736

Disponible: https://www.nucleodoconhecimento.com.br/salud/numero-de-casos 
de diabetes tipo 1 y 2 diagnosticados en Amapá con las variables de género, grupo de edad, sedentarismo, sobrepeso, tabaquismo, entre 2007 y 2012. Los datos de la investigación fueron tomados del departamento informático de SUS, DATASUS (http://datasus.saude.gov.br). La diabetes mellitus tipo 1 y 2 (DM1 y DM2) son enfermedades vinculadas a alteraciones en la producción o en el uso eficiente de insulina. El tabaquismo, así como el sedentarismo y el sobrepeso son factores de riesgo importantes para el desarrollo de DM2. La diabetes mellitus tipo 2 proporciona el desarrollo de varias lesiones nerviosas orgánicas. Además, DM2, a través de su cronicidad, permite el desarrollo de retinopatías, nefropatías y otras condiciones negativas para la salud del individuo.

Palabras clave: Amapá, Diabetes Mellitus, sobrepeso, sedentarismo, fumar.

\section{INTRODUCCIÓN}

La diabetes mellitus es una sucesión de diferentes tipos de trastornos del metabolismo que se caracterizan por causar una alta tasa de azúcar en la sangre, generalmente relacionada con la ausencia de insulina o deficiencia en la absorción de los mismos por el cuerpo (ADA, 2014).

La diabetes tipo 1 (DM1) ocurre cuando el cuerpo ataca las células responsables de producir insulina que conduce a la deficiencia completa de esta hormona en el cuerpo. Ocurre con menos frecuencia porque generalmente está relacionada con factores genéticos. La diabetes tipo 2 (DM2) ocurre cuando el cuerpo no puede absorber la insulina producida en el cuerpo debido a la incapacidad del páncreas para producirla en cantidad suficiente. Esto generalmente ocurre cuando el individuo tiene antecedentes de mala alimentación y estilo de vida sedentario y se adquiere más fácilmente si tiene una tendencia hereditaria a la enfermedad (BRASIL, 2006; MORA et al., 2015).

Los síntomas de la diabetes tipo 1 y 2 incluyen aumento del volumen de orina, exceso de cabeza, aumento del hambre, pérdida de peso, cansancio, cambios de

RC: 66736

Disponible: https://www.nucleodoconhecimento.com.br/salud/numero-de-casos 
humor, hipoglucemia e hiperglucemia. La diabetes tipo 2 puede no presentar síntomas durante varios años, siendo el aumento de la deficiencia de insulina el fusible para la evolución de este tipo (UFRGS, 2016; NOGUEIRA et al., 2015).

Debido a que es una enfermedad con factores genéticos tipo 1 la diabetes tiene como principal factor de riesgo la herencia, mientras que la diabetes tipo 2 además de estos factores, incluye obesidad, presión arterial alta, mala educación alimentaria y avance de la edad (LIMA et al.; 2014)

La prevención de la diabetes tipo 2 está directamente relacionada con una vida con buenos hábitos alimenticios y ejercicios físicos regulares. Debido a que es hereditario y autoinmune, no es cómo prevenir la diabetes tipo 1 (MAGALHÃES et al., 2017).

Debido a que DM1 se caracteriza por una deficiencia completa de insulina, se ha demostrado que la terapia con insulina es el mejor tratamiento. DM2 porque es una incapacidad del cuerpo para producir o absorber suficiente insulina, el tratamiento se dedica a mantener el control glucémico del cuerpo, que incluye tanto medicamentos orales como ejercicios físicos junto con una dieta equilibrada (UFRGS, 2016; SANTOS; FREITAS; PINTO, 2014).

En 2012 el número de casos mundiales de diabetes fue de aproximadamente 200 millones, mientras que los casos en Brasil fueron cercanos a los 10 millones (BRASIL, 2012).

\section{OBJETIVO}

Mostrar el número de casos de diabetes tipo 1 y 2 diagnosticados en Amapá con las variables de género, grupo de edad, sedentarismo, sobrepeso, tabaquismo, entre 2007 y 2012.

RC: 66736

Disponible: https://www.nucleodoconhecimento.com.br/salud/numero-de-casos 


\section{MÉTODO}

Datos tomados del departamento informático de SUS, DATASUS (http://datasus.saude.gov.br), siguiendo los siguientes pasos: en primer lugar, se seleccionó la pestaña "acceso a la información", seguida de la opción "información de salud (TABNET)" poco después de la sub-opción "epidemiológica y morbilidad", luego se accedió al grupo de opciones "Hipertensión y diabetes (HIPERDIA)". Se ha seleccionado el siguiente icono "Hyperdia- Registro y Seguimiento de Hipertensivos y Pacientes Diabéticos - desde 2002" la pestaña "seleccionar la opción o hacer clic en el mapa" y si seleccionó la opción "Amapá", para la recopilación de datos, se seleccionó la opción "sexo" en el campo de fila, en el campo de columna la opción "no activa", y en el campo de contenido la opción "tipo 2 diabetes" , después de que se recogieran datos sobre los "períodos disponibles" entre 2007 y 2012; el mismo período utilizado para todas las demás colecciones. La opción "grupo de edad" se seleccionó en el campo de línea, en el campo de columna la opción "no activo" y en el campo de contenido la opción "tipo 2 diabetes". La opción "sexo" se seleccionó en el campo de fila, en el campo de columna la opción "no activo" y en el campo de contenido el campo "tipo 1 diabetes". La opción de grupo de edad se seleccionó en el campo de fila, en el campo de columna la opción "no activo" y en el campo de contenido la opción "tipo 1 diabetes". La opción "año" se seleccionó en el campo de fila, en el campo de columna la opción "no activo" y en el campo de contenido el campo "tipo 1 diabetes". La opción "año" se seleccionó en el campo de fila, en el campo de columna la opción "no activo" y en el campo de contenido el campo "tipo 2 diabetes". La opción "fumar" se seleccionó en el campo de fila, en el campo de columna la opción "no activo" y en el campo de contenido el campo "tipo 1 diabetes". La opción "fumar" se seleccionó en el campo de fila, en el campo de columna la opción "no activo" y en el campo de contenido la opción "tipo 2 diabetes". Los datos se compilaron dentro de la aplicación Excel, un componente de la suite de Microsoft Corporation Office. La investigación bibliográfica se llevó a cabo en artículos científicos, utilizando computadoras del laboratorio de computación del Instituto Federal de Educación, Ciencia y Tecnología de Amapá, Macapá Campus, ubicada

$\mathrm{RC}: 66736$

Disponible: https://www.nucleodoconhecimento.com.br/salud/numero-de-casos 
en: Rodovia BR 210 KM 3, s/n - Bairro Brasil Novo, Código Postal: 68.909-398, Macapá, Amapá, Brasil.

\section{RESULTADOS}

La Figura 1 muestra el número de casos confirmados de diabetes en el estado de Amapá entre 2007 y 2012. Se han diagnosticado más casos de diabetes tipo 2 que el tipo 1.

La Figura 1 muestra el número de casos confirmados de diabetes en el estado de Amapá entre 2007 y 2012.

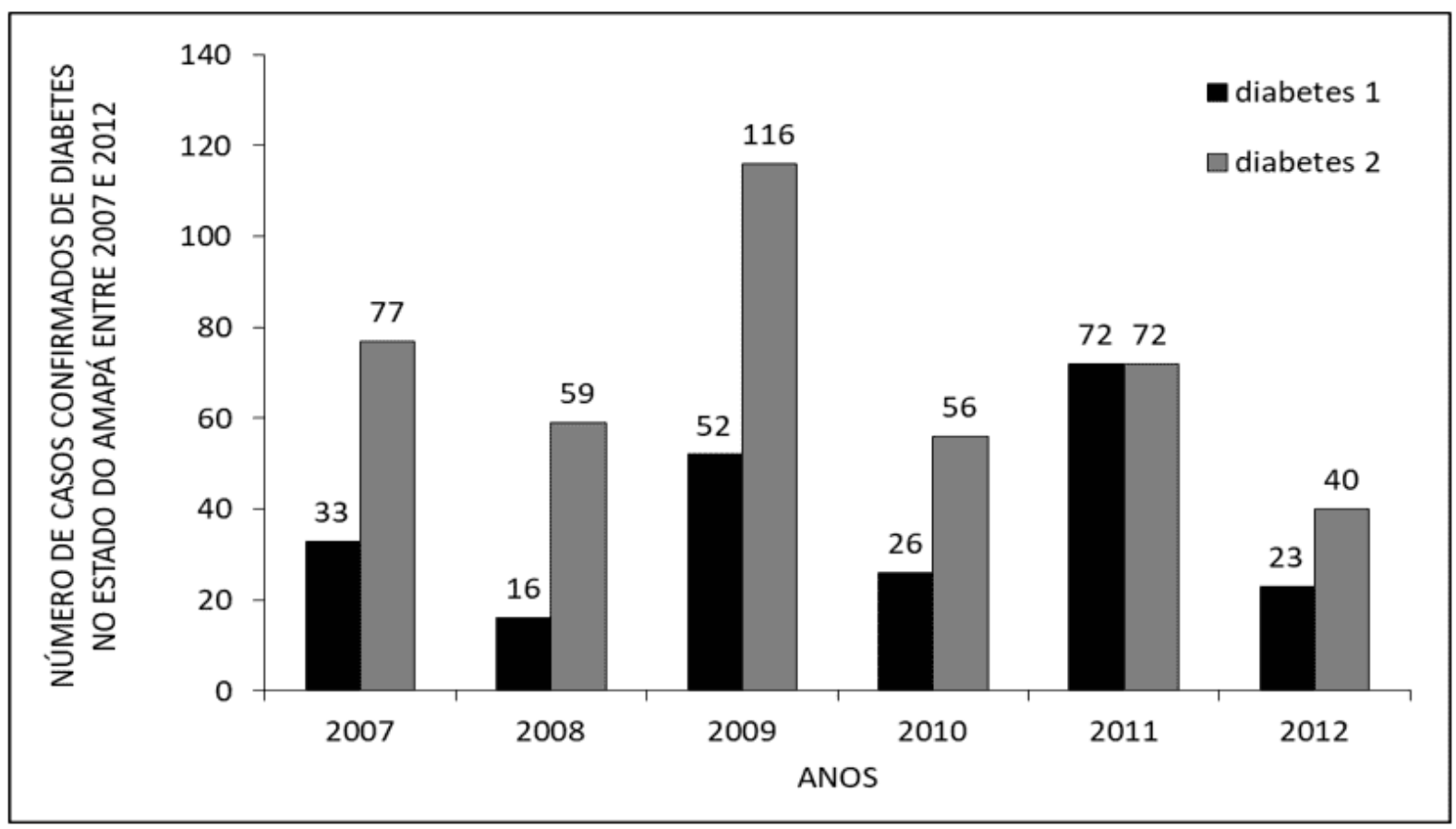

La Figura 2 muestra el número de casos confirmados de diabetes tipo 1 y 2 en el estado de Amapá entre 2007 y 2012 según el género. El mayor número de diagnósticos de diabetes tipo 1 y 2 fue en las mujeres.

RC: 66736

Disponible: https://www.nucleodoconhecimento.com.br/salud/numero-de-casos 
La Figura 2 muestra el número de casos confirmados de diabetes tipo 1 y 2 en el estado de Amapá entre 2007 y 2012 según el género.

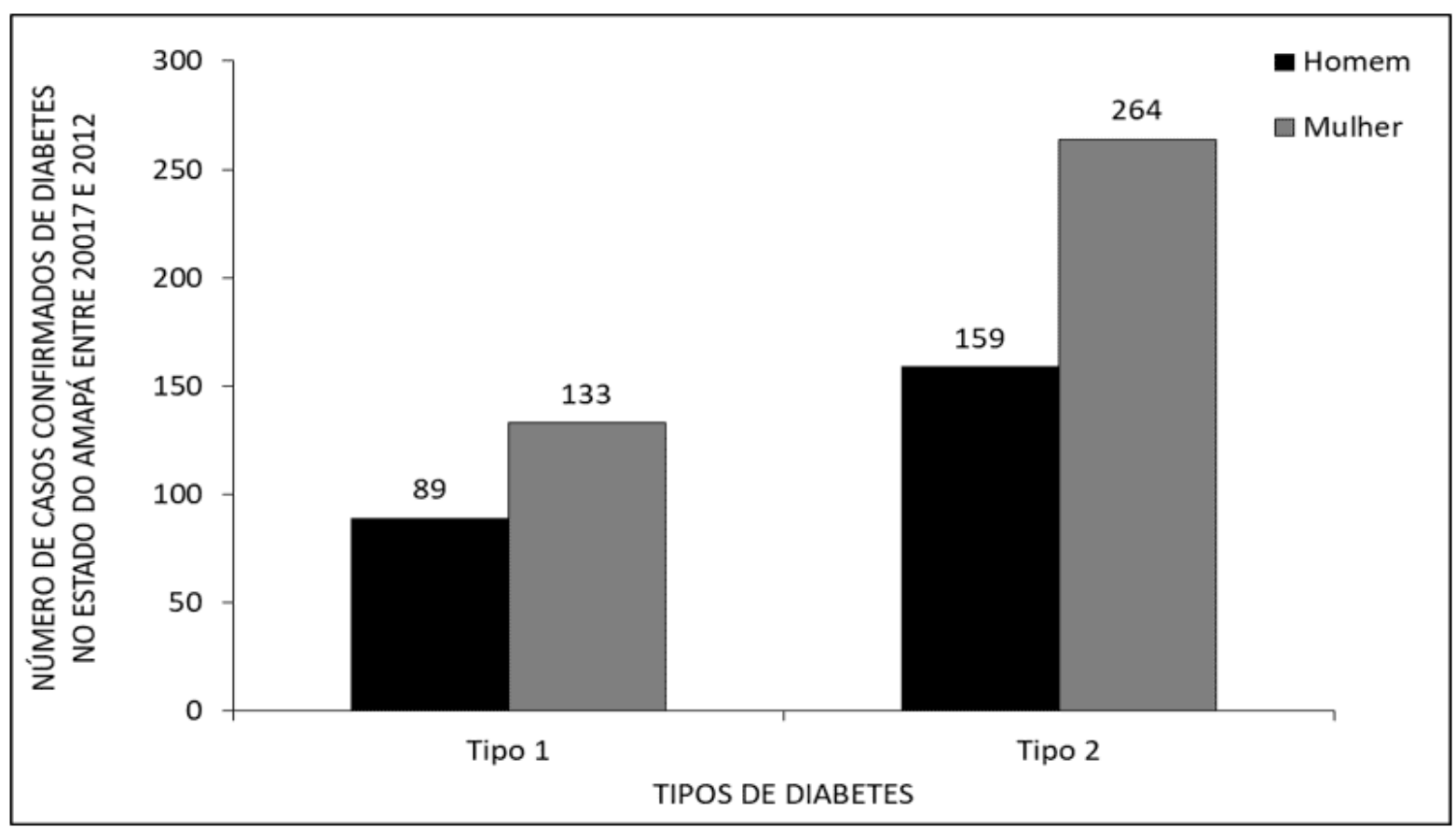

La Figura 3 muestra el número de casos confirmados de diabetes tipo 1 y 2 en el estado de Amapá entre 2007 y 2012 según el grupo de edad. El mayor número de diagnósticos de diabetes tipo 1 y 2 fue de personas de 30 a 59 años, mientras que el más bajo fue de personas de hasta 29 años de edad.

RC: 66736

Disponible: https://www.nucleodoconhecimento.com.br/salud/numero-de-casos 
La Figura 3 muestra el número de casos confirmados de diabetes tipo 1 y 2 en el estado de Amapá entre 2007 y 2012 según el grupo de edad.

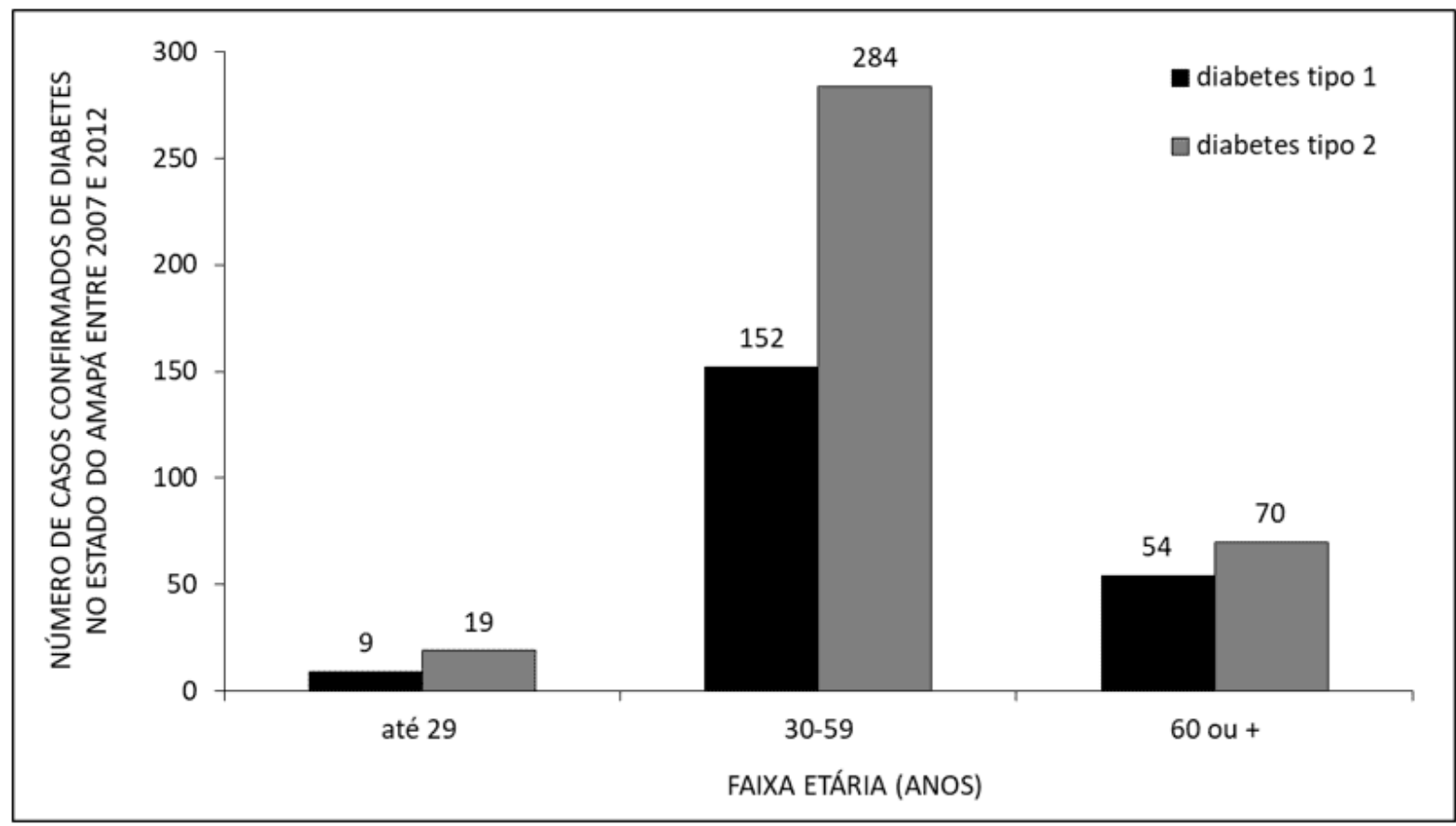

La Figura 4 muestra el número de casos confirmados de diabetes tipo 1 y 2 en el estado de Amapá entre 2007 y 2012 según el peso. El mayor número de casos diagnosticados de diabetes tipo 1 y 2 fue de personas sin sobrepeso.

RC: 66736

Disponible: https://www.nucleodoconhecimento.com.br/salud/numero-de-casos 
La Figura 4 muestra el número de casos confirmados de diabetes tipo 1 y 2 en el estado de Amapá entre 2007 y 2012 según el peso.

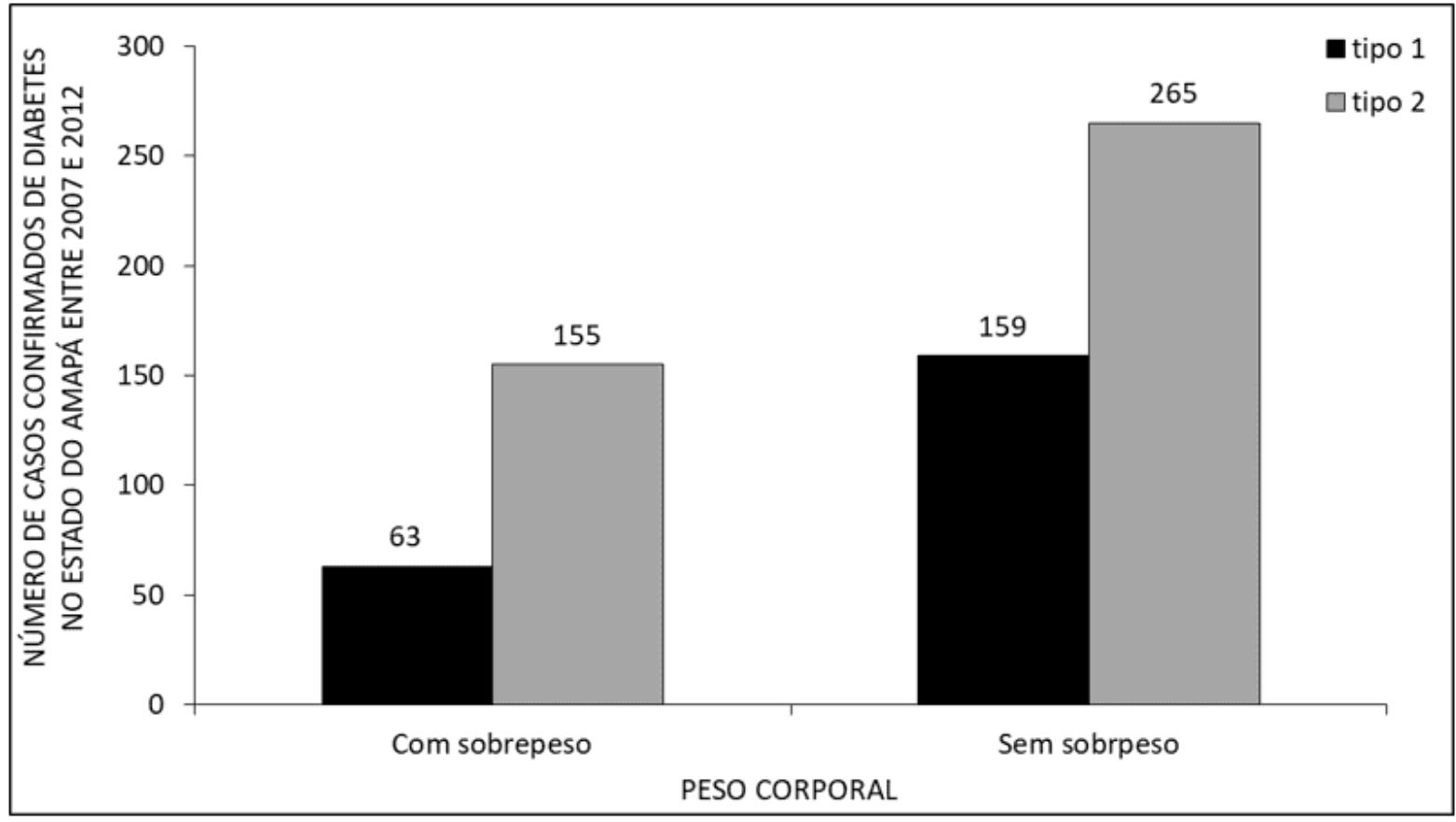

La Figura 5 muestra el número de casos confirmados de diabetes tipo 1 y 2 en el estado de Amapá entre 2007 y 2012 según el estilo de vida sedentario. El mayor número de diagnósticos de diabetes tipo 1 y 2 fue de personas no sedentarias.

RC: 66736

Disponible: https://www.nucleodoconhecimento.com.br/salud/numero-de-casos 
La Figura 5 muestra el número de casos confirmados de diabetes tipo 1 y 2 en el estado de Amapá entre 2007 y 2012 según el estilo de vida sedentario.

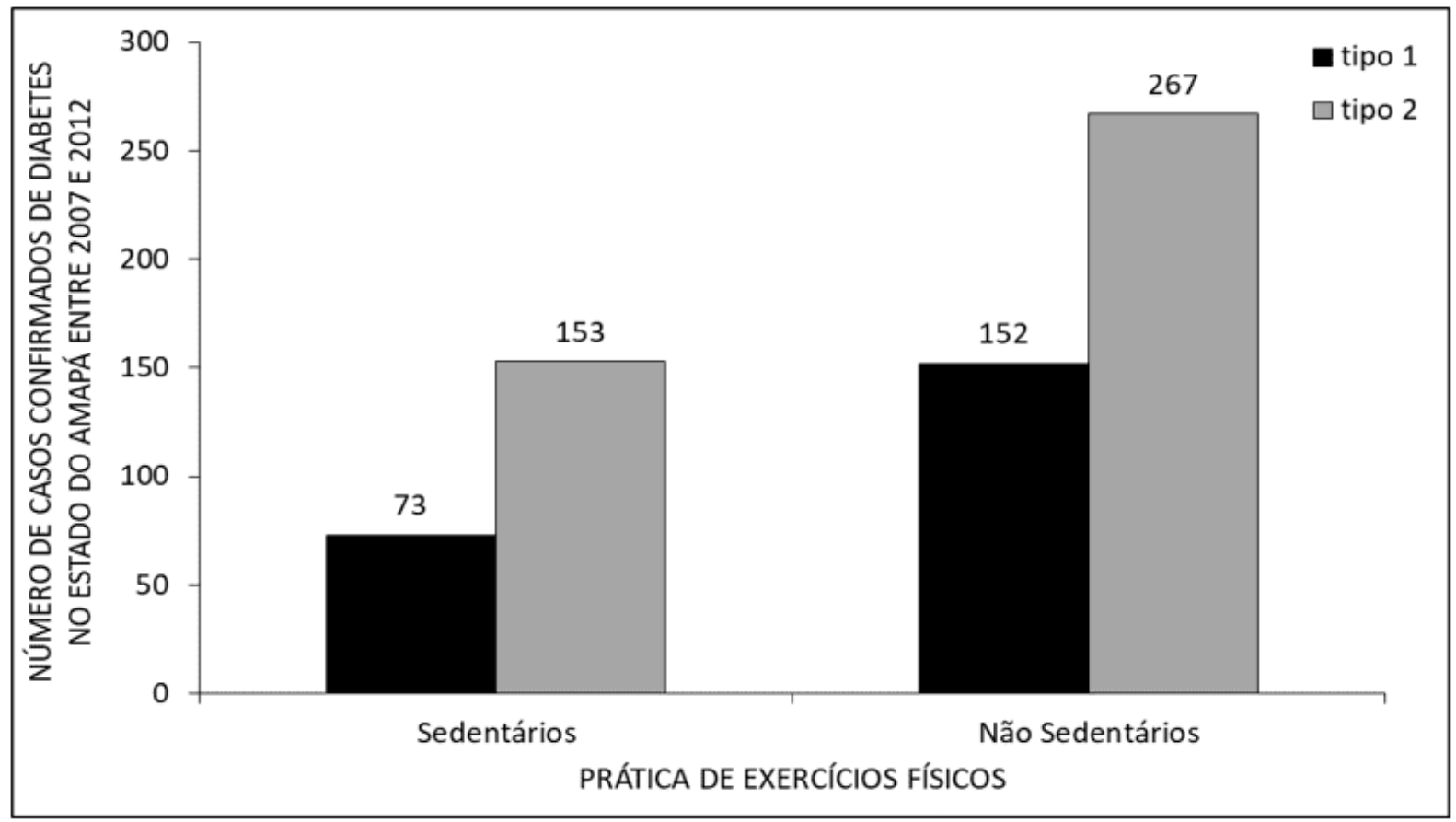

La Figura 6 muestra el número de casos confirmados de diabetes tipo 1 y 2 en el estado de Amapá entre 2007 y 2012 según el tabaquismo. El mayor número de diagnósticos de diabetes tipo 1 y 2 fue de no fumadores.

RC: 66736

Disponible: https://www.nucleodoconhecimento.com.br/salud/numero-de-casos 
La Figura 6 muestra el número de casos confirmados de diabetes tipo 1 y 2 en el estado de Amapá entre 2007 y 2012 según el tabaquismo.

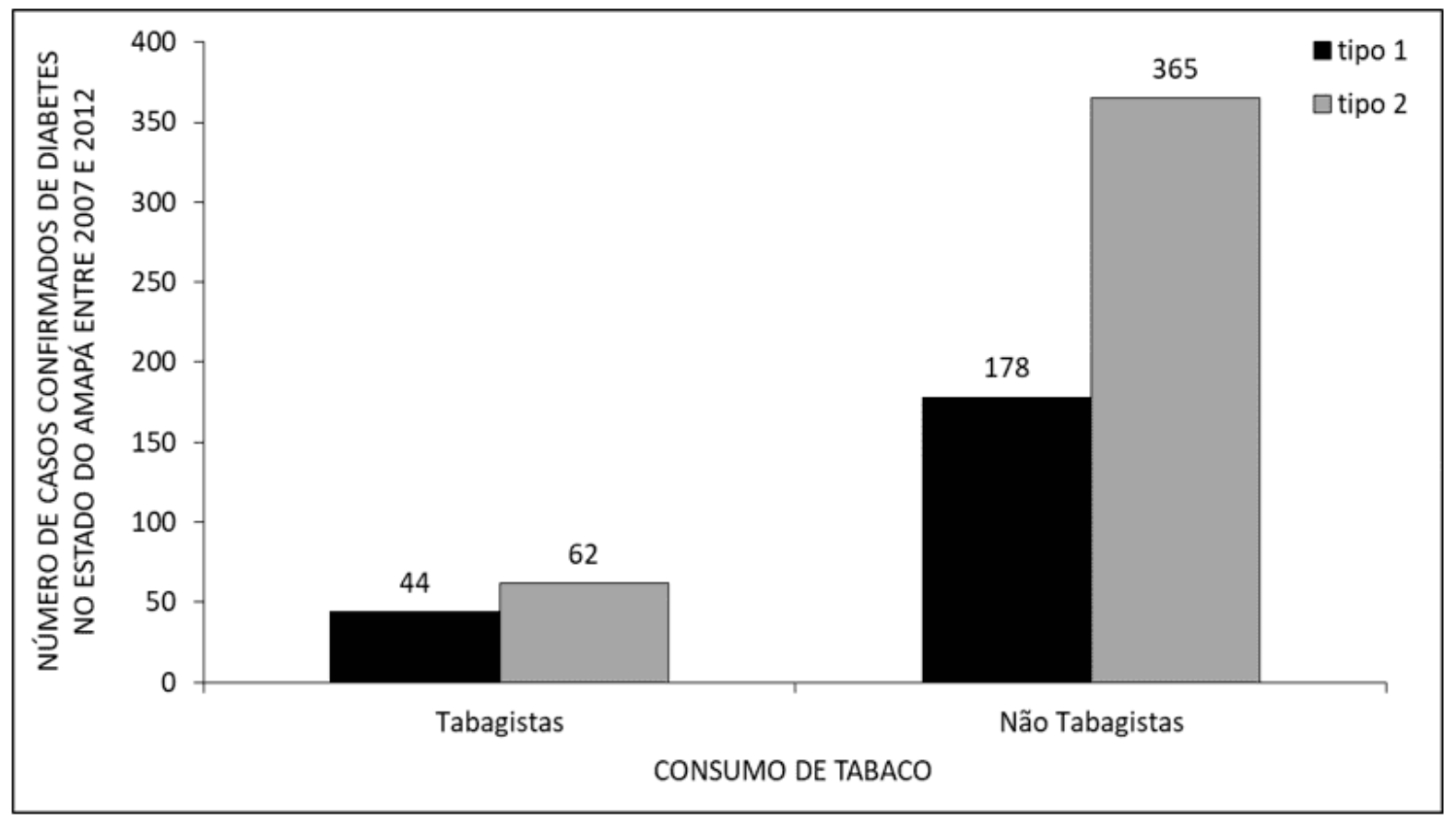

\section{DISCUSIÓN}

La diabetes mellitus tipo 2 (DM2) es una de las principales Enfermedades Crónicas No Transmisibles (ENT), y representa alrededor del $90 \%$ de todos los casos actuales de diabetes (COSTA et al., 2017). Este factor está muy asociado con la prevalencia de la obesidad, ya que este es uno de los principales factores de riesgo para el desarrollo de DM2. El número de pacientes obesos (que tienen DM2) que reciben atención específica y adecuadamente estructurada para que puedan controlar su peso es muy bajo, y, por lo tanto, se observa que este problema constituye un factor de riesgo importante para la salud individual, ya que la obesidad representa un riesgo para el desarrollo de diversas enfermedades, como las enfermedades cardiovasculares, influyendo de manera en gran medida negativa en el control de DM2 (LIMA et al. , 2015).

RC: 66736

Disponible: https://www.nucleodoconhecimento.com.br/salud/numero-de-casos 
Se observa que la prevalencia de la diabetes entre las mujeres es alta, y hay varios factores a analizar para este hallazgo. En un estudio realizado en el municipio de São Leopoldo, en Rio Grande do Sul, se evaluaron numerosos parámetros y variables para este problema. Se observó que la mayor prevalencia de diabetes se produjo entre las mujeres que tienen: edad entre 40 y 49 años, estado civil casado, ingresos por debajo de 1 salario mínimo, 1 a 3 hijos, práctica de tabaquismo, hipertensión arterial sistémica (HAS), y otros factores, como la obesidad, que es el factor de riesgo más importante para DM2 (DIAS-DA-COSTA et al., 2020).

La diabetes mellitus tipo 1 (DM1), también llamada diabetes dependiente de insulina, se presenta con mayor frecuencia en pacientes adolescentes, y su fisiopatología implica la destrucción de las células beta pancreáticas -responsables de la producción de insulina en el cuerpo- y, en consecuencia, llevando al cuerpo a una deficiencia de la producción de insulina, haciendo que el individuo dependa del uso de insulina sintética. DM2 generalmente ocurre después de 30 años, siendo más común en individuos entre 50 y 60 años. (ABREU, 2017). Su fisiopatología está relacionada con la resistencia a la insulina y, por lo tanto, la acción hipoglucémica realizada por esta hormona no se produce adecuadamente, causando un aumento de la producción de glucosa por el hígado, contribuyendo ampliamente con la alta cantidad de insulina a nivel de sangre (BERTONHI y DIAS, 2018).

Aunque, entre 2007 y 2012, el número de casos confirmados de DM1 y DM2 en Amapá fue mayor entre las personas sin sobrepeso, no sedentarios y no fumadores, la asociación entre DM2 y estas condiciones es bien conocida. Una gran proporción de pacientes con diabetes mellitus tipo 2 son obesos o tienen sobrepeso. La práctica del tabaquismo es responsable del aumento exponencial de la posibilidad de que el individuo desarrolle una neoplasia, siendo (de forma aislada) la principal causa de cáncer en todo el mundo. La diabetes tiene un vínculo íntimo con el cáncer, ya que aumenta la posibilidad de desarrollar neoplasias hepáticas, además de cánceres de colon, endometrio, mama y páncreas. Por lo tanto, se observa la predisposición de los fumadores diabéticos a las neoplasias. Además, el estilo de vida sedentario se

$\mathrm{RC}: 66736$

Disponible: https://www.nucleodoconhecimento.com.br/salud/numero-de-casos 
caracteriza como una de las variables a tener en cuenta para el desarrollo de DM2. En un estudio realizado con estudiantes de la Universidad Federal de Ceará (UFC), se observó la vehemente falta de ejercicio físico regular, a veces justificado debido a la falta de motivación y tiempo, además del cansancio proveniente del viaje diario del estudiante. La ausencia de práctica diaria de ejercicios físicos puede promover el sobrepeso (sobrepeso y obesidad), que son factores de riesgo para el desarrollo de DM2 (LIMA et al., 2015; HOCAYEN y MALFATTI, 2010; LIMA et al., 2014).

\section{CONCLUSIÓN}

La diabetes mellitus tipo 1 y 2 (DM1 y DM2) son enfermedades vinculadas a alteraciones en la producción o en el uso eficiente de insulina. DM1 es una enfermedad cuya fisiopatología no se conoce completamente que implica predisposición genética, combinada con factores ambientales. DM2, por otro lado, aunque tiene bases genéticas, está muy cerca del estilo de vida del individuo, y la obesidad, el sobrepeso y el sedentarismo son uno de los principales factores de riesgo para el desarrollo de esta patología.

Se observa que, entre los diabéticos, la prevalencia de las mujeres es muy alta. Factores como la edad entre 40 y 49 años, ingresos por debajo de 1 salario mínimo, estado civil casado, 1 a 3 hijos, práctica de tabaquismo, hipertensión arterial sistémica (HAS), y otros factores, como la obesidad son responsables de este problema.

DM1 ocurre con mayor frecuencia en pacientes adolescentes, y su fisiopatología implica la destrucción de células beta pancreáticas, causando en el cuerpo una deficiencia de la producción de insulina, haciendo que el individuo dependa del uso de insulina sintética. DM2 ocurre con mayor frecuencia después de 30 años, siendo más común en individuos entre 50 y 60 años, y su fisiopatología está relacionada con la resistencia a la insulina y, en vista de esto, la acción hipoglucémica realizada por la insulina no ocurre adecuadamente en el cuerpo, causando, a medida que se

RC: 66736

Disponible: https://www.nucleodoconhecimento.com.br/salud/numero-de-casos 
produce la cronicidad de la enfermedad, una serie de consecuencias negativas para los organismos, con lesiones macro y microvasculares y entre otras.

El tabaquismo, así como el sedentarismo y el sobrepeso son factores de riesgo importantes para el desarrollo de DM2. La diabetes mellitus tipo 2 proporciona el desarrollo de varias lesiones nerviosas orgánicas. Además, DM2, a través de su cronicidad, permite el desarrollo de retinopatías, nefropatías y otras condiciones negativas para la salud del individuo.

\section{REFERENCIAS}

ABREU, L. C. S. Diabetes na Terceira Idade. Revista Científica Multidisciplinar Núcleo do Conhecimento, v. 2, p. 111-131, 2017.

ADA. American Diabetes Association; Diagnosis and Classification of Diabetes Mellitus; Diabetes Care, Volume 37, Supplement 1, January 2014.

BERTONHI, L. G.; DIAS, J. C. R. Diabetes mellitus tipo 2: aspectos clínicos, tratamento e conduta dietoterápica. Revista Ciências Nutricionais Online, v. 2, n. 2, p. 1-10, 2018.

BRASIL. Ministério da Saúde. Secretaria de Atenção à Saúde. Departamento de Atenção Básica. Diabetes Mellitus / Ministério da Saúde, Secretaria de Atenção à Saúde, Departamento de Atenção Básica. - Brasília : Ministério da Saúde, 2006.

BRASIL. Portal Brasil. Diabetes traz consequências graves se não for controlado. 2012. disponível em: <http://www.brasil.gov.br/saude/2012/04/diabetes>. Acessado Em: 19/09/2017.

CORTEZ. D.N; Reis, I.A; Souza, D.A.S; Macedo, M.M.L; Torres H.C Complicações e o tempo de diagnóstico do diabetes mellitus na atenção primária Acta Paulista de Enfermagem, vol. 28, núm. 3, 2015, pp. 250-255.

$\mathrm{RC}: 66736$

Disponible: https://www.nucleodoconhecimento.com.br/salud/numero-de-casos 
COSTA, A. F.; FLOR, L. S.; CAMPOS, M. R.; OLIVEIRA, A. F.; COSTA, M. F. S.; SILVA, R. S.; LOBATO, L. C. P.; SCHRAMM, J. M. A. Carga do diabetes mellitus tipo 2 no Brasil. Cad. Saúde Pública, v. 33, n. 2, p. 1-14, 2017.

DIAS-DA-COSTA, J. S.; SILOCCHI, C.; SCHWENDLER, S. C.; MORIMOTO, T.; MOTTIN, V. H. M.; PANIZ, V. M. V.; BAIRROS, F. S.; OLINTO, M. T. A. Prevalência de diabetes mellitus autorreferido em mulheres e fatores associados: estudo de base populacional em São Leopoldo, Rio Grande do Sul, 2015. Epidemiol. Serv. Saude, v. 29, n. 2, p. 1-12, 2020.

HOCAYEN, P. A. S.; MALFATTI, C. R. M. Tabagismo em pacientes diabéticos: predisposição às doenças crônico-degenerativas e neoplasia. Cinergis, v. 11, n. 2, p. 19-25, 2010.

LIMA, A. C. S.; ARAÚJO, M. F. M.; FREITAS, R. W. J. F.; ZANETTI, M. L.; ALMEIDA, P. C.; DAMASCENO, M. M. C. Fatores de risco para diabetes mellitus tipo 2 em universitários: associação com variáveis sociodemográficas. Rev. LatinoAm. Enfermagem, v. 22, n. 3, p. 484-490, 2014.

LIMA, L. L.; SÁ, A. D.; FIGUEIREDO, A. S.; MUÑOZ, R. L. S. Prevalência de sobrepeso e obesidade em diabéticos tipo 2 atendidos no ambulatório de Endocrinologia de um Hospital Universitário. Rev Soc Bras Clin Med, v. 13, n. 4, p. 251-256, 2015.

MORA, G.P.C.; Abascal I.C.; Sanabria, G. Sobrepeso, obesidad y diabetes mellitus 2 en adolescentes de América Latina en 2000-2010;Revista Cubana de Medicina General Integral. v. 31, n. 3, 217-231, 2015.

SANTOS, M. S, Freitas, N. M; Pinto, F. O; O DIABETES MELLITUS TIPO 1 E TIPO 2 E SUA EVOLUÇÃO NO MUNICÍPIO DE QUISSAMÃ-RJ; Revista Científica Interdisciplinar, vol 1,No 1, 2014.

RC: 66736

Disponible: https://www.nucleodoconhecimento.com.br/salud/numero-de-casos 
UFRGS. RegulaSUS. Diabetes Mellitus. Disponível em: <https://www.ufrgs.br/telessauders/documentos/protocolos_resumos/endocrino_resu mo_diabetes_TSRS_20160324.pdf >.Acesso em: 19/09/2017.

Artículo: Diciembre de 2020.

Aprobado: Diciembre de 2020.

RC: 66736

Disponible: https://www.nucleodoconhecimento.com.br/salud/numero-de-casos 